\title{
Relación entre redes personales y calidad de vida en individuos desmovilizados del conflicto armado colombiano*
}

\section{Personal networks and quality of life in Colombian individuals demobilized from the armed conflict}

Recibido: junio 8 de 2010 | Revisado: septiembre 6 de 2010 | Aceptado: septiembre 15 de 2010

\author{
JOSÉ AMAR-AMAR ** \\ RAIMUNDO ABELLO-LLANOS \\ CAMILO MADARIAGA OROZCO \\ Universidad del Norte, Barranquilla, Colombia \\ José HeRnANDo Ávila-TosCANO*** \\ Universidad del Sinú Elías Bechara Zainúm, \\ Montería, Colombia
}

\section{RESUMEN}

El objetivo de este artículo fue identificar la existencia de relaciones significativas entre los indicadores estructurales y funcionales de las redes personales de individuos desmovilizados y sus niveles de calidad de vida física y psicológica. Se evaluaron 37 individuos residentes en una zona de distensión militar en El Caribe colombiano aplicando el SF-36 y el ASSIS en un estudio correlacional. Los datos se examinaron mediante Análisis de Redes Sociales (ARS) y cálculo estadístico con la $t$ de Student y el coeficiente de correlación de Pearson. Se observaron redes pequeñas, con altos niveles de insatisfacción frente al apoyo social y reducidos promedios de calidad de vida. Se registraron diferencias significativas en la percepción general de apoyo de las redes y la obtención objetiva de ayuda $(p=0.000)$, así como correlaciones entre los niveles de participación social en las redes y la vitalidad psicológica de los individuos.

Palabras clave autores

Redes personales, calidad de vida, apoyo social, desmovilizados.

Palabras clave descriptores

Investigación psicológica, test psicológico, comportamiento social, comportamiento de grupo, relaciones interpersonales, afectividad, voluntad de realización.

Para citar este artículo. Amar-Amar, J., AbelloLlanos, R., Madariaga, O. C. \& Ávila-Toscano, J. H. (2011). Relación entre redes personales y calidad de vida en individuos desmovilizados del conflicto armado colombiano. Universitas Psychologica, 10 (2), $355-369$.

* Artículo de investigación.

** Grupo de Investigaciones en Desarrollo Humano (GIDHUM). Universidad del Norte. Dirección: Universidad del Norte, $\mathrm{Km} 5$ vía Puerto Colombia, A.A. 1569 (Barranquilla, Colombia). E-mails: jamar@uninorte.edu.co; rabello@uninorte.edu.co; cmadaria@uninorte.edu.co

**** Grupo de Investigación en Ciencias Sociales y Humanidades. Correspondencia: Carrera 1W Cl. 38 Juan XXIII. Bloque 5 P2 Of. 03 (Montería, Colombia) Coordinación de investigaciones en Psicología. E-mail: joseavila@unisinu.edu.co

\section{A B S T R A C T}

The Aim of this study was to identify the existence of significant relationships between structural and functional indicators of personal networks of demobilized individuals and their levels of physical and psychological quality of life. We assessed 37 individuals living in an area of military distension in the Colombian Caribbean using the SF-36 and the ASSIS in a correlational study. The data were examined with Social Network Analysis (SNA) and statistical calculation with the Student t and Pearson correlation coefficients. There were small networks, with high levels of dissatisfaction with social support and low indices of quality of life. There were significant differences in the general perception of support networks and obtaining objective help $(p=0.000)$ and correlations between levels of social participation in networks and psychological vitality of individuals.

Key words authors

Personal Networks, Quality Of Life, Social Support, Demobilized.

Key words plus

Psychological Research, Psychological Tests, Social Behaviour, Group Behaviour, Interpersonal Relations, Emotions, Achievement Motivation. 


\section{Introducción}

En la actualidad, el estudio de las redes sociales es uno de los campos más fértiles para la Psicología aplicada. Su relevancia radica en la aplicación de métodos sofisticados para la comprensión de fenómenos sociales, mediante el empleo de datos reticulares que examinan atributos individuales dentro de un contexto de grupo. Una red social es un conjunto definido de actores vinculados mutuamente, por medio de un conjunto de relaciones sociales; dentro de estas redes hay fuertes componentes afectivos y emocionales en los cuales los individuos encuentran protección y apoyo (Montero, 2003).

Comprender las redes sociales implica reconocer el carácter de intercambio que se define en las relaciones de los elementos que las componen, por lo que se las considera como estructuras de intercambio social. Adler de Lomnitz (1998) percibe la red como una estructura cuyos componentes esenciales recaen sobre las atenciones sociales en materia de apoyo, protección y solidaridad, como forma de afrontamiento de las condiciones de desventaja en las que la orientación de unos hacia otros sustenta las prácticas de intercambios (Abello, Madariaga \& Hoyos, 1996); además, el intercambio afectivo e instrumental actúa como mecanismo de fortalecimiento político, económico, laboral y de actividad solidaria de las redes socialmente vulnerables por su condición socioeconómica, el estado de desplazamiento y migración, la influencia de la exclusión social, etc. (Madariaga, Abello \& Sierra, 2003).

El método de evaluación de las redes se denomina Análisis de la Redes Sociales (ARS), considerado como el estudio de la estructura social (Hawe, Webster \& Shiell, 2004), mediante un método cuantitativo que se basa en las regularidades del patrón de relaciones establecidas entre entidades sociales definidas como personas, grupos u organizaciones (Hawe et al., 2004), dado que lo social no se constituye mediante categorías o atributos individuales, sino a través de los vínculos entre los actores aunque los mismos no sostengan relaciones directas (Lozares, 2005). A través del análisis de los datos generados en las redes (análisis reticular), se asume que las características identificadas en las relaciones de la red determinan los comportamientos de los individuos que actúan en ellas, superando la noción de los sistemas sociales como constituidos por individuos con normas y principios individuales o por interacciones diádicas (Trujillo, Mañas \& González-Cabrera, 2010). Sanz Menéndez (2003) por su parte, considera que el ARS define un nuevo paradigma de las Ciencias Sociales que da cuenta de las relaciones y estructuras surgidas en el plano de lo relacional más allá de la definición de atributos de los actores; así mismo, su poder explicativo es importante.

\section{Redes sociales y calidad de vida}

Sluzki (1996) considera que la red social posee carácter salutogénico, pues ofrece herramientas para la conservación de la salud y los resultados de la investigación social aplicada resaltan su importancia sobre las condiciones de bienestar, salud y calidad de vida de los individuos. La pertenencia a la red garantiza mayores posibilidades de afrontamiento de las condiciones difíciles, dado que los intercambios emocionales que fluyen entre los actores, se sustenta en la entrega de soporte socioafectivo que favorece la amortización del impacto de las situaciones desfavorables así como el mejor desarrollo de la calidad de vida.

El estudio de la calidad de vida ha sido un fenómeno que ha venido creciendo desde los años 60 (Verdugo \& Sabeh, 2002), en la actualidad son numerosos los estudios y contextos en los que se aplica (González-Celis \& Padilla, 2006; Palacio, Amar, Madariaga, Llinás \& Contreras, 2007; Palacio \& Madariaga, 2006; Schwartzmann, 2003). Se trata de un concepto que comprende componentes subjetivos y objetivos (Trujillo, Tovar \& Lozano, 2004), relacionados con la percepción que cada individuo posee acerca de su propia vida, los cuales pueden afectar el nivel de calidad de la misma en función de la forma como se presentan dentro de las dinámicas sociales y contextuales, y en el marco de los intereses personales del individuo (VelardeJurado \& Ávila-Figueroa, 2002). 
La red social se relaciona con la salud y la calidad de vida a través de diversos mecanismos como el proporcionamiento de apoyo social (Gallicchio, Hoffman \& Helzlsouer, 2007), manifestado a nivel emocional e instrumental, la influencia entre los actores de la red, el contacto cara a cara (Dekker, 2005; Lahuerta, Borrell, Rodríguez-Sanz, Pérez $\&$ Nebot, 2004), etc. El uso del ARS ha permitido identificar la forma como la estructura de la red influye en la salud o los riesgos para la misma (Christley et al., 2005; Remor, 2002). Robles et al. (2000), por ejemplo, identificaron que el apoyo emocional de las redes de ancianos puede servir como elemento de amortiguamiento de problemáticas psicológicas que afectan la calidad de vida. Herrera y Gracia (2005) señalaron que un pobre nivel de ajuste psicosocial es frecuente entre aquellos individuos que se encuentran aislados del medio comunitario; Rutledge, Matthews, Lui, Stone y Cauley (2003), demostraron el poder de la compañía y el apoyo emocional representado en el matrimonio, dado que las mujeres casadas cuentan con una red social amplia, con un mejor estado general en su bienestar y una menor probabilidad de muerte, es decir, su calidad de vida aumentaba en materia de longevidad y satisfacción personal. Una perspectiva similar ofrecen algunos estudios desarrollados con población japonesa, en los cuales se ha identificado que la falta de redes sociales para hombres y mujeres constituye un fuerte predictor de mortalidad (Iwasaki et al., 2002).

La evidencia investigativa tiende a resaltar los efectos positivos que tienen los apoyos sociales de las redes, así como el desempeño significativo de roles dentro del contexto social sobre la calidad de vida de los individuos, al igual que los efectos adversos que puede tener sobre la calidad de vida la ausencia de las favorabilidades del apoyo social de la red. Frente a esto, Ávila-Toscano (2009) plantea que la dinámica de las relaciones sostenidas en la red puede favorecer al bienestar, pero también comporta la existencia de debilidades que pueden afectar de manera negativa la salud mental y física de los individuos, de allí que se requiera abordar la estructura y funcionalidad social desde perspectivas integradoras y lo suficientemente amplias como para comprender la existencia de ambas caras de la moneda.

\section{Conflicto armado, desmovilización, redes sociales y calidad de vida}

La literatura sobre redes sociales permite identificar investigaciones que relacionan los movimientos poblacionales, la despoblación y las migraciones con la temática de las redes, éste es un campo ampliamente explorado y fructífero, en el que existen hallazgos relevantes frente al tema. Maya Jariego (2004) ha demostrado que la reubicación de las comunidades inmigradas en zonas de recepción, afecta las condiciones de adaptación psicológica e integración social lo que influye sobre los procesos de conformación de las redes. La gran mayoría de las personas sometidas a migraciones ve modificadas sus redes sociales producto del desplazamiento (Maya Jariego, 2002), pese a ello, deben trabajar en procura de la integración con la red social lo cual requiere del desarrollo de procesos de asimilación, modificación y acomodación dentro de los parámetros sociales que regulan el funcionamiento de la red. En algunas ocasiones, la llegada de miembros nuevos al contexto de la red puede desatar reacciones problemáticas como sucede con los individuos desmovilizados que retornan o llegan por vez primera a los núcleos comunitarios. La realidad contextual hacia la condición de desmovilizado puede conllevar a que la red se muestre hermética o viceversa, afectando el proceso de adaptación. Sin embargo, las posibilidades de adaptación, aún en condiciones complejas, son potenciadas por las mismas redes, puesto que los resultados en materia de adecuación a su dinámica y al desarrollo de inclusión social puede verse asociado al grado de pertenencia que desarrolla el individuo por las redes comunitarias. Ahora bien, en los fenómenos de desplazamiento, retorno a núcleos comunitarios y migración, los individuos reciben de sus redes sociales apoyo emocional, instrumental e informativo, poniendo de primer plano muestras de solidaridad y cooperación entre los elementos de la red; sin embargo, tras la movilización hacia la zona receptora y el necesario proceso de reajuste en 
el sistema de apoyo de origen, los individuos antes de establecer cualquier vínculo de auxilio realizan un análisis de la disponibilidad de los vínculos de manera que pueden escoger sus formas preferentes de apoyo social (García, Martínez \& Albar, 2002).

Por otra parte, los fenómenos migratorios - incluida la desmovilización - pueden presentarse como una consecuencia de los movimientos armados en determinadas regiones, de manera que la violencia política es un factor que cobra protagonismo en ello. Diversos estudios han abordado el impacto de la violencia sobre las redes sociales. Aguilar y Molina (2004) desarrollaron un estudio en Sarajevo en el cual analizaron las relaciones existentes entre las condiciones de identidad étnica en adolescentes y el establecimiento de redes personales; los resultados mostraron que los jóvenes con un discurso cosmopolita, no etnicista presentaban mejores niveles de interacción en las redes personales, lo que revela la importancia de la ampliación del sistema de relaciones e intercambios sociales. Palacio y Madariaga (2006) identificaron, en una población colombiana, que entre las comunidades de individuos desplazados por la violencia predominan relaciones que tienden al encapsulamiento y apartamiento de las vinculaciones con los demás elementos del contexto social, producto de la estructuración de lazos fuertes que surgen como respuesta a la inseguridad producida por el estado de vulneración y la pobreza. El encapsulamiento generado en tales redes se refleja en un elevado nivel de confianza en la red familiar y una desconfianza general por lo comunitario en el orden de lo macro (Dallo, 2005).

La desmovilización por su parte, implica la migración y relocación de los actores generando una fracturación de las redes previamente establecidas al interior de los grupos armados. Estos efectos sociales negativos inmediatos conllevan a un pesimismo en el desmovilizado frente a la integración con la red, que dificulta la construcción de arraigo producto de la reubicación permanente o de la tendencia a sentirse excluidos. No es raro que en estos casos se dé una propensión por conservar un apego emocional con los hábitos y costumbres de las redes construidas en los ambientes alzados en armas, manifestada en el recuerdo y la deseabilidad de dinámicas anteriores de relación, al experimentar frente a las mismas mayor proximidad social percibida lo que las hace preferiblemente recordadas (Molina, McCarty, Aguilar \& Rota, 2008).

La reubicación del desmovilizado comporta una gama de fenómenos sociales relacionados con una imagen criticada y desfavorable de quienes llegan de la guerra; este fenómeno conduce a procesos de marginación, exclusión y estigmatización que se solapan entre problemáticas inherentes a los procesos de relocación de los individuos (Sluzki, 1996), además se puede relacionar con los estudiados efectos de la segregación (Takács, 2007) en los grupos en conflicto.

En este estudio, se analizan las características estructurales y funcionales de un grupo de individuos desmovilizados, con el fin de identificar componentes que develen las formas particulares de relación de los desmovilizados a través de sus redes personales, así como de reconocer de una manera más cercana y objetiva sus formas particulares de ofrecimiento de apoyo e intercambios sociales y materiales, como medios para la superación de las adversidades determinando la relación de tales prácticas con la calidad de vida.

\section{Método}

\section{Muestra}

Estudio correlacional de corte transversal y enfoque cuantitativo en el que la población del estudio la constituyen 550 individuos desmovilizados del conflicto armado colombiano, ubicados en zona urbana (290 participantes) y rural (260 participantes) del municipio de Tierralta (Córdoba, Colombia, Sur América). Se seleccionaron 37 individuos debidamente identificados como desmovilizados por los entes designados por la Presidencia de la República de Colombia para tal fin. Los individuos fueron escogidos mediante muestreo intencional (Namakforoosh, 2005) entre aquellos sujetos identificados y certificados como desmovilizados por la Alta Consejería Presidencial para la Reintegración. Se consideró apto a todo individuo desmovilizado en condición grupal que voluntariamente aceptó 
participar en el estudio; la escogencia se realizó indistintamente de su género y fue requisito que su edad, al momento de la desmovilización, hubiera sido superior a los 18 años y que tuviera un tiempo mayor a seis meses de vivir en la zona urbana donde se desarrolló el estudio, con el fin de garantizar la existencia de una red de socialización estructurada en la zona de acomodación.

\section{Instrumentos}

La medición de la calidad de vida se realizó con el Cuestionario SF-36, el cual consta de 36 ítems que componen 8 dimensiones para evaluar la calidad de vida percibida en relación con la salud física (Función Física, Rol físico, Dolor corporal, Salud General) y psicológica (Vitalidad, Función Social, Rol Emocional y Salud mental). Entre más alta sea la puntuación mejor es el rendimiento general. Los estudios de salud física y mental de los factores del instrumento han identificado entre un 80 y un 85 $\%$ de fiabilidad de la varianza entre las escalas de medición del SF-36.

La información relacionada con la red social fue recogida mediante el Arizona Social Support Interview Schedule (ASSIS) (Barrera, 1980), el cual es un instrumento que facilita la generación de nombres de personas que conforman la red social de los participantes evaluados, estableciendo además el tipo de apoyo social percibido por los mismos (Palacio \& Madariaga, 2006). El ASSIS permite identificar la red social en función de la descripción de seis dimensiones específicas de apoyo como lo son: Sentimientos personales, Ayuda material, Consejo, Retroalimentación positiva, Asistencia física y Participación social. El ASSIS muestra una confiabilidad test-retest de 0.88 tras reevaluar en un periodo de tres días, y de 0.70 tras la repetición de la prueba en un período de un mes; así mismo, la consistencia interna ofrece un rango de puntuaciones entre 0.32 y 0.52 (López et al., 2007).

\section{Procedimiento}

Inicialmente los participantes de la muestra fueron informados respecto a las dimensiones, límites y finalidades del estudio con el objetivo de que su participación estuviera ajustada a un claro conocimiento de su rol como sujetos de investigación. Posteriormente, se obtuvo la firma del consentimiento informado y se procedió a completar el SF-36 de forma heteroaplicada. A continuación, se realizó la aplicación del ASSIS, mediante el establecimiento de una entrevista semiestructurada. La aplicación de los instrumentos fue controlada mediante consistencia.

Una vez recogida la información, fue procesada en una base de datos creada para la identificación de los indicadores estructurales de las redes mediante el software Ucinet 6.181 (Borgatti, Everett \& Freeman, 2002), basado en el Análisis centrado en un solo actor (Verd Pericás \& Martí Olivé, 1999), a partir del cual se logró la evaluación de redes egocéntricas a través de las cuales se analizaron las relaciones que poseen los actores dentro de la red (Burt, Jannotta \& Mahoney, 1998). Se generaron visualizaciones (grafos) de las redes empleando Netdraw, herramienta gráfica contenida en Ucinet. Los aspectos funcionales y los indicadores de calidad de vida fueron analizados a través del SPSS 18.0 Statistics, con el cual se realizó un análisis descriptivo para el cálculo de los promedios de los indicadores de red y las dimensiones de calidad de vida; posteriormente, se aplicó la $t$ de Student para muestras relacionadas, con el fin de comparar las medias de los indicadores funcionales de acuerdo al reporte general de apoyo descrito en la red frente al nivel de apoyo recibido en el último mes. Por último, se aplicó el coeficiente de correlación de Pearson $(r)$, para establecer posibles relaciones entre las características funcionales y estructurales con los niveles de calidad de vida de los participantes.

\section{Resultados}

\section{Características demográficas de los participantes}

La muestra quedó conformada por 37 individuos de los cuales 2 fueron mujeres constituyendo el 5,4 $\%$ mientras que los hombres $(n=35)$ representa- 
ron el 96,4\%. Esta notoria diferencia en función del sexo, es un patrón que se presenta en todo el territorio colombiano en donde solamente el 14,1 $\%$ de las personas en proceso de reintegración son mujeres (3.878 mujeres de 31.199 desmovilizados activos en proceso de reintegración) con una proporción de 1:7 frente a los hombres.

Los individuos evaluados presentaron edades comprendidas entre los 21 y los 60 años con una media de 31,73 años (DT $=7,621)$. Los evaluados tenían una media de tiempo de desmovilización de 3,86 años $(D T=0,481)$. En su mayoría sostenían una relación conyugal siendo la unión de libre el estado civil más frecuente $(81,1 \%, n=30)$ mientras que la unión matrimonial alcanzó el 2,7 \% (n $=1$ ); así mismo, 5,4\% de los individuos expresó ser soltero $(n=2)$ y el $10,8 \%$ separado $(n=4)$.

\section{Características estructurales de las redes}

Las redes establecidas se caracterizan por ser pequeñas al contar con un número de miembros que no supera los 14 individuos, siendo en su mayoría hombres. En las 37 redes evaluadas se reportó un total de 433 alteri (integrantes de la red) de los cuales 283 son hombres y 150 mujeres con una proporción de 1 mujer a 2.89 hombres por red personal. Las edades de los alteri oscilaron entre un mínimo de 24 y un máximo de 48 años ( $\mu=$ $37,47 ; D T=6,085)$. El número de individuos desmovilizados reportados como alteri en las 37 redes alcanzó un valor de 69 personas (15,9\%), siendo un promedio considerablemente bajo.

En general se puede describir una red personal de un desmovilizado de la muestra como una estructura social constituida por un promedio de 11,7 alteri, 7,04 de ellos hombres y 4,05 mujeres, con una edad promedio en alteri de 37,47 años y con 1,86 miembros desmovilizados. El tipo de relación predominante es la familiar seguida de las amistades cercanas y, en raras ocasiones, se integran redes con compadres o personas poco conocidas. Se trata de redes construidas con personas que viven generalmente en el mismo barrio, muy cerca de la propia casa, y con los cuales se establece diálogo de forma frecuente.
Por su parte, los diferentes valores de los indicadores estructurales (centralidad, cercanía e intermediación) obtenidos mediante ARS se encuentran contenidos en la Tabla 1, donde se aprecian todos los valores, tanto brutos como normalizados, de cada participante.

Los resultados de la Tabla 1 permiten observar que en relación a la Centralidad el 48,6 \% de la muestra $(n=18)$ presenta un nivel bajo, $19 \%$ medio $(n=7)$ y $32,4 \%$ alto $(n=12)$. Estos datos indican un promedio considerable de individuos desmovilizados con baja centralidad (Figura 1). La cercanía señala valores similares dado que el 51.4 $\%$ de los evaluados $(n=19)$ presenta un nivel bajo, $13,5 \%$ un nivel medio $(n=5)$ y $35,1 \%$ un nivel alto $(n=13)$ esto demuestra un predominio de reducidos niveles de contactos sociales.

Los individuos que aparecen con promedios elevados de cercanía lo hacen con un valor equivalente a 100,000 lo cual indica que la intermediación en la red equivale a cero (0), pues se reporta un conocimiento y vinculación total entre todos los integrantes por lo que no se requieren intermediarios para el flujo informativo, a este fenómeno se le conoce como redes de estrella con centralización cero ( 0 ) en las cuales se indica la ausencia de nodos claramente centrales (Velásquez \& Aguilar, 2005), fenómeno que se evidencia en 15 de las 37 redes evaluadas; ejemplos de estas se aprecian en las Figuras 2 y 3 en las cuales el tamaño de todos los nodos es igual, lo cual indica que todos presentan el mismo número de contactos sin que existan intermediarios para las vinculaciones. La baja intermediación se registra en el 51,4\% de la muestra.

\section{Características funcionales de las redes}

En la muestra sobresalen los reportes de alta retroalimentación positiva (RP), señalada con el 74,36 \% de los alteri, seguidamente se observa la dimensión ayuda material (AM) como la segunda más reseñada con un 69,28\%, de esta forma, los intercambios instrumentales representa uno de los aspectos de mayor relevancia (Madariaga et al., 2003; Palacio \& Madariaga, 2006b). La dimensión de Consejo (C), es la tercera que recibe mayor señalamiento 
RELACIÓN ENTRE REDES PERSONALES Y CALIDAD DE VIDA EN INDIVIDUOS DESMOVILIZADOS...

TABLA 1

Valores de los indicadores estructurales brutos y normalizados en las redes personales evaluadas

\begin{tabular}{|c|c|c|c|c|c|c|c|}
\hline \multicolumn{8}{|c|}{ Medidas estructurales (de centralidad) de las redes personales evaluadas } \\
\hline \multirow[b]{2}{*}{ Sujeto } & \multicolumn{3}{|c|}{ Centralidad } & \multicolumn{2}{|c|}{ Cercanía } & \multicolumn{2}{|c|}{ Intermediación } \\
\hline & Degree & SD & NmrDegree $^{1}$ & InCloseness $^{2}$ & OutCloseness $^{3}$ & Betwenness & nBetweenness $^{4}$ \\
\hline 1 & 8,000 & 1,265 & 88,889 & 81,221 & 80,549 & 2,600 & 3,611 \\
\hline 2 & 4,500 & 1,658 & 64,286 & 74,628 & 74,451 & 2,750 & 6,548 \\
\hline 3 & 5,143 & 0,990 & 85,714 & 83,061 & 83,401 & 1,429 & 4,762 \\
\hline 4 & 6,889 & 1,449 & 86,111 & 88,078 & 87,331 & 1,333 & 2,381 \\
\hline 5 & 5,818 & 2,757 & 58,182 & 70,891 & 70,300 & 4,818 & 5,354 \\
\hline 6 & 6,333 & 2,779 & 57,576 & 69,927 & 69,506 & 5,417 & 4,924 \\
\hline 7 & 8,182 & 1,749 & 81,818 & 82,522 & 83,362 & 2,364 & 2,626 \\
\hline 8 & 9,091 & 1,379 & 90,909 & 10,909 & 92,837 & 0,455 & 1,010 \\
\hline 9 & 10,000 & 3,338 & 76,923 & 83,418 & 83,774 & 3,071 & 1,969 \\
\hline 10 & 10,429 & 2,945 & 80,220 & 84,811 & 84,682 & 2,786 & 1,786 \\
\hline 11 & 10,857 & 1,767 & 83,516 & 83,709 & 82,237 & 2,929 & 1,877 \\
\hline 12 & 8,857 & 2,030 & 68,132 & 75,994 & 75,717 & 4,429 & 2,839 \\
\hline 13 & 12,286 & 0,881 & 94,505 & 92,105 & 92,150 & 1,214 & 0,778 \\
\hline 14 & 8,857 & 2,642 & 68,132 & 75,766 & 75,679 & 4,714 & 3,022 \\
\hline 15 & 13,733 & 0,573 & 98,095 & 96,009 & 95,722 & 0,667 & 0,366 \\
\hline 16 & 14,000 & 0,000 & 100,000 & 95,722 & 96,320 & 0,667 & 0,366 \\
\hline 17 & 9,857 & 2,695 & 75,824 & 78,870 & 77,404 & 4,143 & 2,656 \\
\hline 18 & 9,846 & 1,791 & 82,051 & 84,769 & 84,869 & 2,385 & 1,807 \\
\hline 19 & 10,429 & 2,382 & 80,220 & 80,585 & 80,106 & 3,643 & 2,335 \\
\hline 20 & 8,000 & 2,572 & 66,667 & 72,720 & 71,470 & 5,154 & 3,904 \\
\hline 21 & 11,077 & 1,071 & 92,308 & 90,093 & 91,047 & 1,385 & 1,049 \\
\hline 22 & 13,000 & 0,000 & 100,000 & 13,000 & 100,000 & 0,000 & 0,000 \\
\hline 23 & 13,000 & 0,000 & 100,000 & 13,000 & 100,000 & 0,000 & 0,000 \\
\hline 24 & 9,000 & 0,000 & 100,000 & 90,727 & 92,352 & 1,000 & 1,389 \\
\hline 25 & 16,923 & 2,814 & 70,513 & 100,000 & 100,000 & 0,000 & 0,000 \\
\hline 26 & 13,846 & 2,032 & 57,692 & 100,000 & 100,000 & 0,000 & 0,000 \\
\hline 27 & 18,154 & 3,060 & 75,641 & 100,000 & 100,000 & 0,000 & 0,000 \\
\hline 28 & 14,923 & 2,730 & 62,179 & 100,000 & 100,000 & 0,000 & 0,000 \\
\hline 29 & 14,462 & 2,373 & 60,256 & 100,000 & 100,000 & 0,000 & 0,000 \\
\hline
\end{tabular}


José Amar-Amar, Raimundo Abello-llanos, Camilo Madariaga Orozco, José Hernando Ávila-Toscano

\begin{tabular}{|c|c|c|c|c|c|c|c|}
\hline \multicolumn{8}{|c|}{ Medidas estructurales (de centralidad) de las redes personales evaluadas } \\
\hline \multirow[b]{2}{*}{ Sujeto } & \multicolumn{3}{|c|}{ Centralidad } & \multicolumn{2}{|c|}{ Cercanía } & \multicolumn{2}{|c|}{ Intermediación } \\
\hline & Degree & SD & NmrDegree $^{1}$ & InCloseness $^{2}$ & OutCloseness $^{3}$ & Betwenness & nBetweenness ${ }^{4}$ \\
\hline 30 & 15,385 & 2,704 & 64,103 & 100,000 & 100,000 & 0,000 & 0,000 \\
\hline 31 & 14,993 & 1,236 & 53,333 & 100,000 & 100,000 & 0,000 & 0,000 \\
\hline 32 & 19,231 & 2,722 & 80,128 & 100,000 & 100,000 & 0,000 & 0,000 \\
\hline 33 & 17,692 & 3,073 & 73,718 & 100,000 & 100,000 & 0,000 & 0,000 \\
\hline 34 & 16,143 & 2,326 & 62,088 & 100,000 & 100,000 & 0,000 & 0,000 \\
\hline 35 & 14,667 & 2,560 & 66,667 & 100,000 & 100,000 & 0,000 & 0,000 \\
\hline 36 & 18,000 & 2,563 & 69,231 & 100,000 & 100,000 & 0,000 & 0,000 \\
\hline 37 & 14,500 & 2,291 & 65,909 & 100,000 & 100,000 & 0,000 & 0,000 \\
\hline
\end{tabular}

Nota. ${ }^{1}$ Grado nodal normalizado, ${ }^{2}$ Cercanía de entrada, ${ }^{3}$ Cercanía de salida, ${ }^{4}$ Intermediación normalizada.

Fuente: elaboración propia.

Figura 1

Grafo de baja centralidad Sujeto 4 (Hombre)

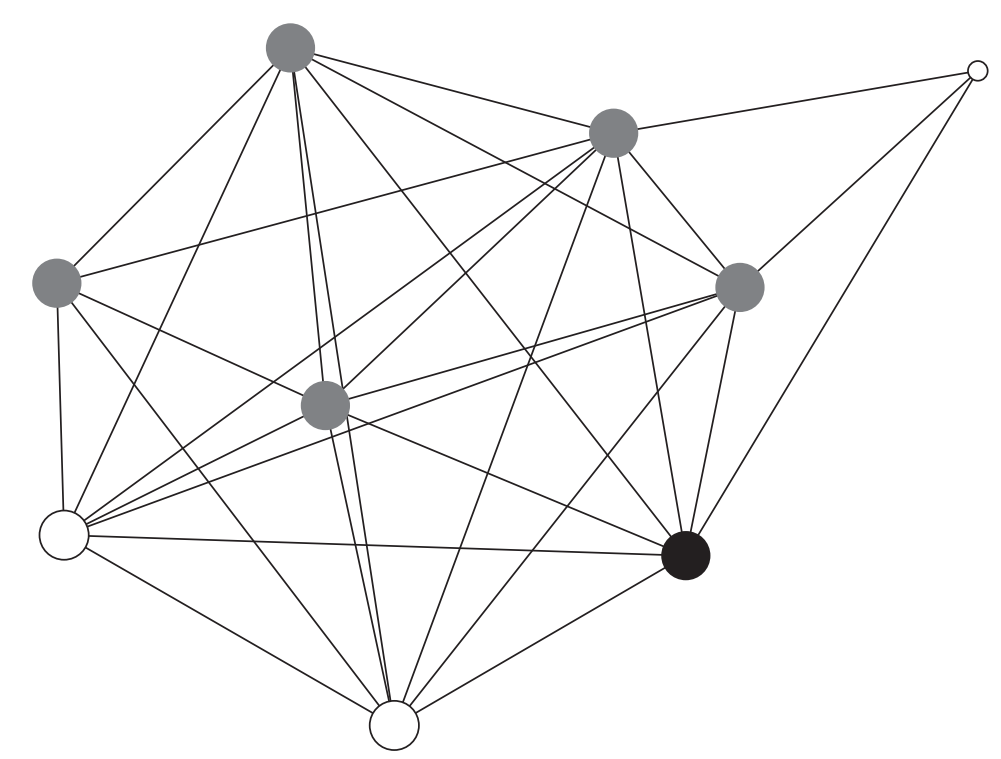

Valores calculados con ARS: NmrDegree = 86,111; nCloseness = 87,331; nBetweenness = 2,381

Nota. Negro: Ego; Gris: Familiar; Blanco: Conocido; Tamaño: Centralidad.

Fuente: elaboración propia. 
Figura 2

Grafo de estrella Sujeto 27 (Hombre)

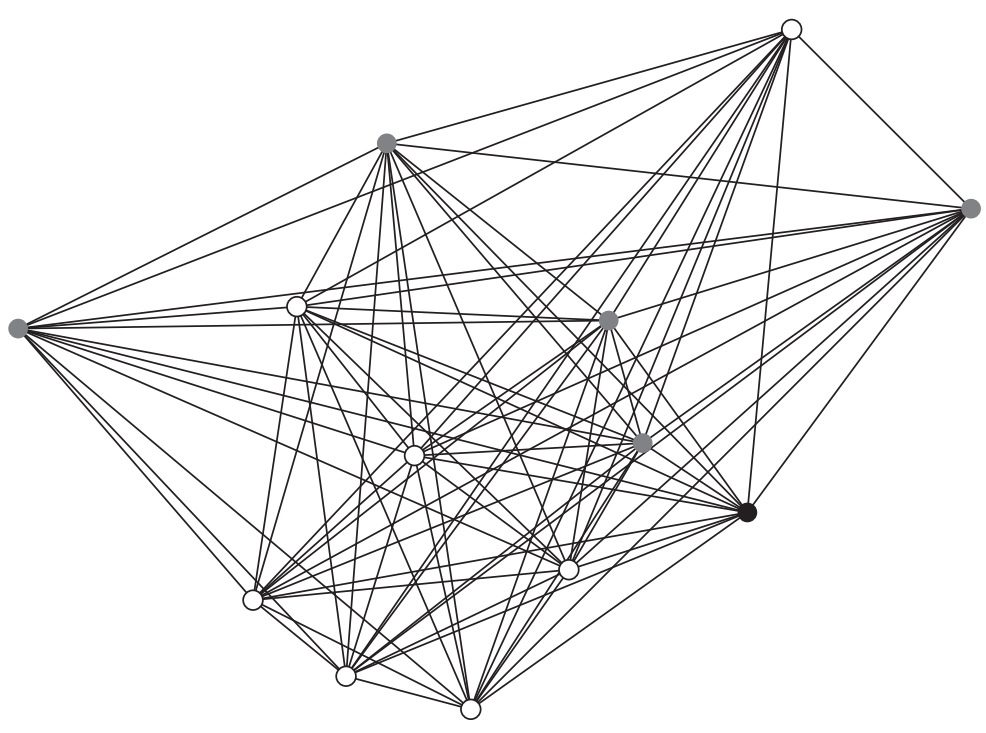

Valores calculados con ARS: NmrDegree $=75,641 ;$ nCloseness = 100,000; nBetweenness = 0,000. Nota: Negro: Ego; Gris: Familiar; Blanco: Conocido.

Fuente: elaboración propia.

FIGURA 3

Grafo de estrella Sujeto 37 (Hombre)

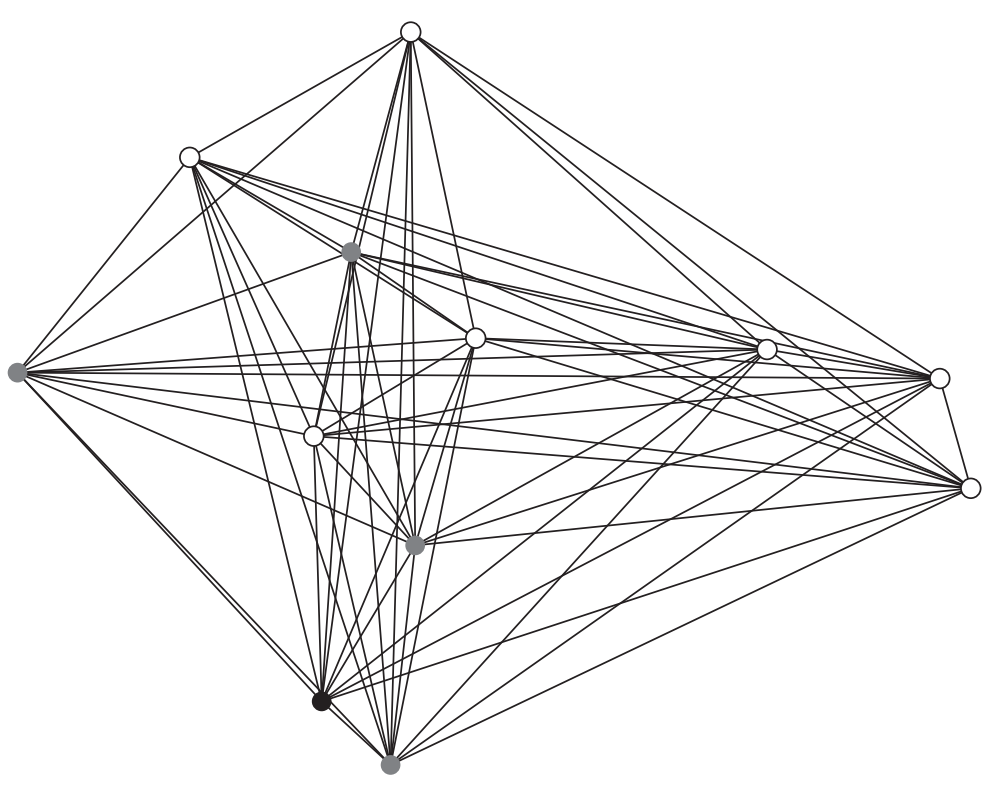

Valores calculados con ARS: NmrDegree =65,909; Cercanía nCloseness = 100,000; Betweenness = 0,000. Nota. Negro: Ego; Gris: Familiar; Blanco: Buen amigo.

Fuente: elaboración propia. 
como elemento de interacción $(57,04 \%)$ mientras que la participación social (PS) y la asistencia física (AF) muestran promedios similares alcanzando valores del 56,58 y $53,58 \%$, respectivamente, por último, los sentimientos privados (SP) son señalados como la dimensión de menor referencia $(51,73 \%)$, es decir, se expresa con menor medida los asuntos íntimos a los alteri.

Los resultados de las dimensiones funcionales fueron comparados de acuerdo a los criterios de percepción de la ayuda en cada dimensión y la recepción de ayuda objetiva en el último mes mediante la $t$ de Student para muestras relacionadas, la cual muestra diferencias significativas en todas las dimensiones de apoyo social evaluadas al compararlas con la recepción de ayuda en el último mes (Sentimientos privados $t=7,403 ; p=0.000$, Ayuda material $t=9,484 ; p=0.000$, Consejo $t=$ 5,$197 ; p=0.000$, Retroalimentación positiva $t=$ 5,$965 ; p=0.000$, Asistencia física $t=5,258 ; p=$ 0.000 , Participación social $t=4,460 ; p=0.000$ ), esto indica que la ayuda recientemente recibida es significativamente menor a la ayuda general esperada. La menor recepción de ayuda en el último mes coincide con los resultados del nivel de satisfacción que los individuos evaluados tienen del apoyo que reciben de sus redes personales. El indicador funcional con la cual se muestran más insatisfechos es la ayuda material (AM) (61\%) aún cuando ésta es señalada como una de las formas de apoyo que más reciben los desmovilizados; la retroalimentación positiva (RP) $(51 \%)$ muestra niveles elevados de insatisfacción al igual que la dimensión de consejo (C) (40\%), la satisfacción con éste indicador parece ser poco común entre los hombres $(r=-0.530 p<0.01)$ y se presenta además con un número menor de alteri $(r=-0.359$ $p<0.05)$, es decir, parecen ser más satisfechos los egos de redes pequeñas. La participación social (PS) es la única dimensión en la cual los desmovilizados se muestran mayormente satisfechos (58\%) con la ayuda recibida. Los sentimientos privados (SP) muestran insatisfacción y correlaciona con aquellos alteri que comparten la vivienda con el participante $\mathrm{o}$ actor principal de la red $(r=0.340$ $p<0.05)$. La asistencia física (AF) muestra resul- tados particulares al ser señalada como el indicador funcional con mayor nivel medio de satisfacción hacia el cual los individuos expresan conformidad. La AF presenta además relaciones significativas con los alteri que son familiares $(r=0.352 p<$ $0.05)$ a la par que correlaciona negativamente con los conocidos de vista $(r=-0.357 p<0.05)$.

\section{Calidad de vida e indicadores de redes sociales}

El panorama de la calidad de vida es considerablemente reducido, mostrando medias para la dimensión física y psicológica por debajo de los puntos establecidos como criterio de oro (Vilaguta et al., 2005). De acuerdo con la calidad de vida física, la dimensión con menor rendimiento es Salud General (SG) $(\mu=55,5)$ mientras que el promedio más alto de rendimiento lo obtiene la Función Física (FF) $(\mu=88,6)$. La calidad de vida psicológica identificó un mayor nivel de rendimiento en comparación con la física, siendo muy cercano a la puntuación criterio los valores de la dimensión Salud Mental (SM) $(\mu=76,5)$, mientras que la dimensión Vitalidad (V) se encuentra por encima de la media en un nivel muy alto $(\mu=88,8)$, entre tanto, las dimensiones Rol Emocional (RE) $(\mu=$ $86,3)$ y Función Social (FS) $(\mu=88,3)$ están por debajo de la media.

La medición de posibles relaciones de la calidad de vida con los indicadores estructurales, permitió observar que las variaciones conjuntas existentes entre las variables no ofrecen resultados significativos de manera que no hay relaciones estadísticas importantes entre los dos conjuntos de variables. Las dimensiones de apoyo social de las redes, por su parte, se correlacionaron con los indicadores estructurales, en este procedimiento se observó que los altos niveles de centralidad se relacionan con niveles elevados de Participación social $(r=0.356$ $p=0.031)$, mayor liberación de Sentimientos privados $(r=0.336 p=0.042)$ y mayor posibilidad de recepción de Ayuda de tipo instrumental $(r=$ $0.410 p=0.012$ ) Así mismo, entre más central es el participante en las redes personales mayores son las posibilidades de acceder a Retroalimentación 
positiva $(r=0.637 p=0.000)$ y a Asistencia física $(r=0.504 p=0.001)$. Entre tanto, la dimensión de Consejo es la única que no correlaciona significativamente con ninguno de los indicadores estructurales calculados.

Por último, las dimensiones de calidad vida fueron cruzadas con los indicadores funcionales de las redes sociales, pero los valores no muestran relaciones estadísticamente significativas a excepción de la dimensión de Vitalidad (V) que se asocia positivamente con el indicador de Participación social (PS) $(r=0.488 p<0.01)$.

\section{Discusión}

Entre los resultados del estudio resalta el tamaño de las redes, las cuales se caracterizan por poseer un número reducido de miembros y por centrarse en las relaciones con familiares. En general, entre las poblaciones vulnerables es común que la familia sea la principal fuente de soporte y que por ende constituyan en amplio número las redes personales, diversos estudios (Abello et al., 1996; Abello, Madariaga \& Sierra, 1999; Palacio \& Madariaga, 2006) han mostrado a la familia como una proveedora de soporte indispensable para la superación de las necesidades, pues el apoyo familiar sirve como un sustento para el desarrollo individual y la calidad de vida (Cox, 2005).

Un resultado particular del estudio consiste en la reducida presencia de miembros desmovilizados en las redes personales, lo cual contrasta con los resultados de otras investigaciones (Aguilar \& Molina, 2004; Palacio \& Madariaga, 2006), en donde la mayoría de los alteri comparten la condición racial, ideológica o política de los egos. La baja conexidad entre desmovilizados puede afectar negativamente la posibilidad de construir una identidad como grupo social, es posible que esta ausencia de integración esté relacionada con el rompimiento de los lazos sociales conformados al interior del grupo ilegal, así como con la falta de identificación con el significado social de desmovilizado, producto de la estigmatización que experimenta este grupo humano, lo cual puede conducir al rechazo que obstaculiza la integración con otros desmovilizados por lo que es esperado un alejamiento de tales actores (Carter \& Feld, 2004). Ahora bien, la tendencia de los individuos a conformar redes sociales encapsuladas merece especial atención. Esta realidad de la estructura cerrada de la red sin duda repercute en los medios de acceso a recursos y en las posibilidades de afrontamiento (Palacios-Espinoza, Pulido \& Montaña, 2009); así mismo, disminuye la obtención de los mismos lo cual dificulta lograr un nivel funcional de integración y la adquisición de alternativas para el afrontamiento de la vida y el mejoramiento de su calidad, lo cual exige el reconocimiento por parte de los nodos de la necesidad de integración con los demás elementos que conforman redes de soporte más amplias y con el medio comunitario en general. Nuestros resultados refuerzan esta posición al mostrar a los desmovilizados como sujetos íntimamente ligados a redes cerradas y con poca flexibilidad para acceder a lo comunitario y para entenderlo como un mecanismo de desarrollo grupal; para el desmovilizado la unión con sujetos similares es una tarea de desarrollo, pues ello permite la creación de un sentimiento de compañía, que lleva a sentir mayor impulso por la organización, participación y el trabajo (Madariaga, Gallardo, Salas \& Santamaría, 2001).

Entre tanto, los indicadores estructurales y funcionales de las redes muestran un nivel bajo de rendimiento, lo cual puede asociarse a dificultades para lograr influencia positiva sobre los demás. Los niveles bajos de cercanía e intermediación descubren un escenario de pobreza en la obtención de apoyo y recursos sociales necesarios para la adaptación y el afrontamiento de las dificultades. Un promedio importante de las redes estudiadas obtienen un valor de intermediación equivalente a cero (redes de estrella), lo que indica que se trata de redes en donde todos los miembros se conocen de forma directa impidiendo la posibilidad de contar con nodos que cumplan el rol de intermediarios para el establecimiento de nuevos vínculos dentro de la red. Por su parte, parece haber una disonancia entre los niveles generales de recursos que consideran recibir de sus alteri y el nivel de apoyo obtenido recientemente. En este 
sentido, se presenta un elevado nivel de demanda de obtención de recursos materiales y emocionales, lo cual se asume como una necesidad evidente de compañía social como medio de superación de las necesidades de los individuos evaluados, aunque tales demandas muestran un sesgo de las relaciones hacia los lazos fuertes (Molina, Ruiz \& Teves, 2005) representados esencialmente por la familia.

El fin fundamental del estudio se basaba en comprobar si existía relación entre la calidad de vida y los indicadores estructurales y funcionales de las redes personales, sin embargo, este fenómeno se identificó parcialmente dado que los indicadores estructurales no mostraron relación alguna con la calidad de vida mientras que de los indicadores funcionales solo la Participación Social se asoció positivamente con la Vitalidad. La ausencia de relación entre los indicadores estructurales y la calidad de vida se asocia con los datos hallados por Palacio y Madariaga (2006), para quienes tampoco hubo relación entre tales variables, lo cual parece resaltar que las asociaciones específicas entre ambos constructos se concentran en determinados indicadores de tipo funcional. Algunos autores han considerado que esta disparidad se debe a que es más fácil hallar correlaciones cuando se evalúan dimensiones subjetivas de apoyo que frente a las características cuantitativas de la red (Cohen \& Willis, 1985). Entre tanto, el apoyo social y el nivel de significado general que los sujetos le dan, depende de variables individuales a partir de las cuales las personas consideran si los recursos que se obtienen de la red son o no pertinentes, ello haría que diversas formas de vinculación o intercambio no sean percibidos como medios de soporte social (Madariaga \& Sierra, 2000) y que, en consecuencia, el juicio de la calidad de vida varíe ante la no consideración de contar con más medios de apoyo que los percibidos (Sagrestano, Feldman, Killingsworth, Woo \& Dunkel-Schetter, 1999).

A pesar de las moderadas relaciones entre calidad de vida y redes personales, este estudio ha permitido obtener indicadores importantes acerca de las redes de socialización de los desmovilizados y de sus niveles de rendimiento físico y psicológico, dentro de contextos caracterizados por las condiciones de marginalidad, pobreza y exclusión. Los datos refuerzan la idea de las redes de personas pobres y en condiciones de exclusión que se repliegan, conformando estructuras encapsuladas o cerradas lo cual, a su vez, muestra concordancia con los bajos niveles de calidad de vida que expresan los participantes del estudio.

El ajuste psicosocial logrado por los desmovilizados tiende a ser reducido y esto coincide con un juicio negativo de la mayoría de los indicadores relacionados con su calidad de vida, lo cual debilita también su nivel de integración social y comunitaria. Para Palacio y Sabatier (2006) la construcción de comunidad es un fenómeno que experimenta dificultades centradas en la forma como se distribuyen los recursos y la falta de confianza, de este modo, los individuos que viven en condiciones de pobreza y no logran la estructuración de redes funcionales tienen que enfrentarse a la ausencia de medios adecuados para la obtención de recursos necesarios para supervivir. En las redes de los desmovilizados, se ha visto una tendencia a la conformación de relaciones cerradas, sin duda esto opera como elemento que reduce las posibilidades de desarrollo y de rompimiento de mecanismos tradicionales de supervivencia que han ido aprendiendo desde su proceso de desmovilización en el desarrollo de procesos de reintegración que, pese a sus esfuerzos, no han dejado de lado el matiz asistencialista que sustenta y refuerza las demandas elevadas de ayuda material.

Los círculos de pobreza traen consigo diversidad de elementos que afectan los niveles de desarrollo físico y mental, ante lo cual las poblaciones pobres deben trabajar mediante sus redes, para el ofrecimiento de mejores mecanismos para la supervivencia y conservación de la salud. Para ello, es necesario superar la tendencia al encapsulamiento social mediante la configuración de redes más complejas y con probabilidades mayores de obtener un número más importante de recursos, superando la noción asistencialista de la ayuda para transformarla en una idea de movimiento continuo, dinámica y de gestión sociocomunitaria incesante. 


\section{Referencias}

Abello, R., Madariaga, C. \& Hoyos, O. (1996). Redes sociales: un mecanismo de supervivencia en sectores de pobreza. Investigación y Desarrollo, 4, 73-89.

Abello, R., Madariaga, C. \& Sierra, O. (1999). Relationship between the social networks and the family dynamics of low-income working women. Journal of Community Psychology, 27 (3), 243-255.

Adler de Lomnitz, L. (1998). Redes sociales, cultura y poder. Ensayos de antropología latinoamericana. México: Flacso.

Aguilar, C. \& Molina, J. L. (2004). Identidad étnica y redes personales entre jóvenes en Sarajevo. Araucaria, 5 (12), 164-176.

Ávila-Toscano, J. H. (2009). Redes sociales, generación de apoyo social ante la pobreza y calidad de vida. Revista Iberoamericana de Psicología: Ciencia y Tecnología, 2 (2), 65-73.

Barrera, M. (1980). A method for the assessment of social support networks in community survey research. Connections, 3, 8-13.

Borgatti, S. P., Everett, M. G. \& Freeman, L. C. (2002). Ucinet for Windows [Software for Social Network Analysis]. Harvard, MA: Analytic Technologies.

Burt, R., Jannotta, J. E. \& Mahoney, J. T. (1998). Personality correlates in structural holes. Social Networks, 20 (1), 63-87.

Carter, W. C. \& Feld, S. L. (2004). Principles relating social regard to size and density of personal networks, with applications to stigma. Social Networks, 26, 323-329.

Christley, R. M., Pinchbeck, G. L., Bowers, R. G., Clancy, D., French, N. P., Bennett, R. \& Turner, J. (2005). Infection in social networks: Using network analysis to identify high-risk individuals. American Journal of Epidemiology, 162 (10), 1024-1031.

Cohen, S. \& Wills, T.A. (1985). Stress, social support and buffering hypothesis. Psychological Bulletin, 98, 310-357.

Cox, L. (2005). Examining the role of social network intervention as an integral component of community-based, family-focused practice. Journal of Child and Family Studies, 14 (3), 443-454.

Dallo, J. M. (2005). Redes informales: una oportunidad en el diseño del programa de protección social en
Nicaragua. AGER. Revista de Estudios sobre Despoblación y Desarrollo Rural, 4, 27-61.

Dekker, A. (2005). Conceptual distance in social network analysis. Journal of Social Structure, 6 (3). Recuperado el 15 de julio de 2008, de http://www. cmu.edu/joss/content/articles/volume6/dekker/ index.html

Gallicchio, L., Hoffman, S. \& Helzlsouer, K. (2007). The relationship between gender, social support, and health- related quality of life in a communitybased study in Washington County, Maryland. Quality of Life Research, 16, 777-786.

García, M. Martínez, M. \& Albar, M. (2002). La elección de fuentes de apoyo social entre inmigrantes. Psicothema, 14 (2), 369-374.

González-Celis, A. \& Padilla, A. (2006). Calidad de vida y estrategias de afrontamiento ante problemas y enfermedades en ancianos de ciudad de México. Universitas Psicológica, 5 (3), 501-509.

Hawe, P., Webster, C. \& Shiell, A. (2004). A glossary of terms for navigating the field of social network analysis. Journal Epidemiology Community Health, 58, 971-975.

Herrera, J. \& Gracia, E. (2005). Redes sociales de apoyo y ajuste biopsicosocial en la vejez: un análisis comparativo en los contextos comunitario y residencial. Intervención Psicosocial, 14 (1), 41-50.

Iwasaki, M., Otani, T., Sunaga, R., Miyazaki, H., Xiao, L., Wang, N. et al. (2002). Social networks and mortality based on the Komo-Ise cohort study in Japan. International Journal of Epidemiology, 31, 1208-1218.

Lahuerta, C., Borrell, C., Rodríguez-Sanz, M., Pérez, K. $\&$ Nebot, M. (2004). La influencia de la red social en la salud mental de la población anciana. Gaceta Sanitaria, 18 (2), 83-91.

López, I., Álvarez, S., Lorence, B., Jiménez, L., Hidalgo, M. \& Sánchez, J. (2007). Evaluación del apoyo social mediante la escala ASSIS: descripción y resultados en una muestra de madres en situación de riesgo psicosocial. Intervención Psicosocial, 16 (3), 323-337.

Lozares, C. (2005). Valores, campos y capitales sociales. REDES. Revista Hispana para el Análisis de Redes Sociales, 4 (2). Recuperado el 19 de julio de 2008, de http://revistaredes.rediris.es 
Madariaga, C. \& Sierra, O. (2000). Redes sociales y pobreza. Psicología desde El Caribe, 5, 127-156.

Madariaga, C., Gallardo, L. D., Salas, F. \& Santamaría, E. (2001). Violencia política y sus efectos en la identidad psicosocial de los niños desplazados. El caso de La Cangrejera. Psicología desde El Caribe, 10, 88-106.

Madariaga, C., Abello, R. \& Sierra. O. (2003). Redes sociales, infancia, familia y sociedad. Barranquilla: Ediciones Uninorte.

Maya Jariego, I. (2002). Tipos de redes personales de los inmigrantes y adaptación psicológica. Redes. Revista Hispana para el Análisis de Redes Sociales, 4 (1). Recuperado el 18 de julio de 2008, de http:// revista-redes.rediris.es

Maya Jariego, I. (2004). La formación de comunidades de inmigrantes: desplazamiento en cadena y contexto de recepción. Araucaria, 5 (12), 83-91.

Molina, J. (2004). La ciencia de las redes. Apuntes de Ciencia y Tecnología, 11, 36-42.

Molina, J., Ruiz, A. \& Teves, L. (2005). Localizando geográficamente las redes personales. REDES, Revista Hispana para el Análisis de Redes Sociales, 8 (5). Recuperado el 15 de mayo de 2009, de http:// revista-redes.rediris.es

Molina, J., McCarty, C., Aguilar, C. \& Rota, L. (2008). La estructura social de la memoria. En Lozares, C. (Ed.), Interacción, redes sociales y ciencias cognitivas (Capítulo VII). Granada: Editorial Comares S.L.

Montero, M. (2003). Teoría y práctica de la Psicología Comunitaria. La tensión entre comunidad y sociedad. Buenos Aires: Paidós.

Namakforoosh, M. N. (2005). Metodología de la investigación (2aed.). México: Limusa.

Palacio, J. \& Sabatier, C. (2002). Impacto psicológico de la violencia política en Colombia. Barranquilla: Ediciones Uninorte.

Palacio, J. \& Madariaga, C. (2006). Lazos predominantes en las redes sociales personales de desplazados por violencia política. Investigación y Desarrollo, 14 (1), 86-119.

Palacio, J., Amar, J., Madariaga, C., Llinás, H. \& Contreras, K. (2007). La calidad de vida en salud en los menores trabajadores de Toluviejo (Sucre). Investigación y Desarrollo, 15 (2), 366-393.
Palacios-Espinoza, X., Pulido, S. \& Montaña, J. (2009). Estrategias desarrolladas por los niños para afrontar el castigo: un dialogo entre la Psicología y la teoría de redes sociales. Universitas Psychologica, 8 (2), 471-486.

Remor, E. (2002). Apoyo social y calidad de vida en la infección por el VIH. Atención primaria, 30 (3), 143-149.

Robles, L., Rizo Curiel, G., Camarena, L., Cervantes, L., Gómez, M. \& González, M. (2000). Redes y apoyo social en ancianos enfermos de escasos recursos en Guadalajara, México. Cuadernos de Saúde Pública, 16 (2). Recuperado el 22 de febrero de 2010, de http://www.scielosp.org/scielo.php?script $=$ sci arttext\&pid=S0102-311X2000000200026

Rutledge, T., Matthews, K., Lui, L., Stone, K. L. \& Cauley, J. (2003). Social networks and marital status predict mortality in older women: Prospective evidence from the Study of Osteoporotic Fractures (SOF). Psychosomatic Medicine, 65, 688-694.

Sagrestano, L., Feldman, P., Killingsworth, C. H., Woo, G. \& Dunkel-Schetter, C. H. (1999). Ethnicity and social support during pregnancy. American Journal of Community Psychology, 27 (6), 869-898.

Sanz Menéndez, J. (2003). Análisis de redes sociales: o cómo representar las estructuras sociales subyacentes. Apuntes de Ciencia y Tecnología, 7, 20-29.

Schwartzmann, L. (2003). Calidad de vida relacionada con la salud: aspectos conceptuales. Ciencia y Enfermería, 9 (2), 9-21.

Sluzki, C. (1996). La red social: frontera de la práctica sistémica. Barcelona: Gedisa.

Takács, K. (2007). Effects of network segregation in intergroup conflict: An experimental analysis. Connections, 27 (2), 59-76. Recuperado el 18 de junio de 2009, de http://www.insna.org/Connections-Web/Volume27-2/Takacs.pdf

Trujillo, S. Tovar, C. \& Lozano, M. (2004). Formulación de un modelo teórico de la calidad de la vida desde la Psicología. Universitas Psychologica, 3 (1), 89-98.

Trujillo, H., Mañas, F. \& González-Cabrera, J. (2010). Evaluación de la potencia explicativa de los grafos de redes sociales clandestinas con UciNet y NetDraw. Universitas Psychologica, 9 (1), 67-78. 
Velarde-Jurado, E. \& Ávila-Figueroa, C. (2002). Evaluación de la calidad de vida. Salud Pública de México, 44 (4), 349-361.

Velásquez, A. \& Aguilar, N. (2005). Manual introductorio al análisis de redes sociales. Medidas de centralidad. México: Universidad Nacional Autónoma de México.

Verd Pericás, J. M. \& Martí Olivé, J. (1999). Muestreo y recogida de datos en el análisis de redes sociales. Qüestiió, 23 (3), 507-524.
Verdugo, M. A. \& Sabeh, E. N. (2002). Evaluación de la percepción de calidad de vida en la infancia. Psicothema, 14 (1), 86-91.

Vilaguta, G., Ferrera, M., Rajmil, L., Rebollo, P., Permanyer-Miralda, G., Quintana, J. et al. (2005). El Cuestionario de Salud SF-36 español: una década de experiencia y nuevos desarrollos. Gaceta Sanitaria, 19 (2), 135-50. 
